\title{
Research Article \\ Dynamic Properties of a Differential-Algebraic Biological Economic System
}

\author{
Hongyang Zhang and Chunrui Zhang \\ Department of Mathematics, Northeast Forestry University, Harbin 150040, China \\ Correspondence should be addressed to Chunrui Zhang, math@nefu.edu.cn
}

Received 9 August 2011; Accepted 4 October 2011

Academic Editor: Wan-Tong Li

Copyright (c) 2012 H. Zhang and C. Zhang. This is an open access article distributed under the Creative Commons Attribution License, which permits unrestricted use, distribution, and reproduction in any medium, provided the original work is properly cited.

We analyze a differential-algebraic biological economic system with time delay. The model has two different Holling functional responses. By considering time delay as bifurcation parameter, we find that there exists stability switches when delay varies, and the Hopf bifurcation occurs when delay passes through a sequence of critical values. Furthermore, we also consider the stability and direction of the Hopf bifurcation by applying the normal form theory and the center manifold theorem. Finally, using Matlab software, we do some numerical simulations to illustrate the effectiveness of our results.

\section{Introduction}

Recently, dynamics of differential-algebraic equations have become a subject of intense research activities because differential-algebraic equation models can describe many kinds of practical systems such as power system, biological economic system, and singular preypredator economic model $[1,2]$. The dynamic relationship between predators and their preys with harvesting has long been and will continue to be one of the dominant themes in both ecology and mathematical ecology due to its universal existence and importance. Harvesting has a strong impact on the dynamic evolution of a population. Depending on the nature of applied harvesting strategy, the long-run stationary density of the population may be significantly smaller than the long-run stationary density of a population in the absence of harvesting. Time delays of one type or another have been incorporated into mathematical models of population dynamics due to maturation time, capturing time, or other reasons. In general, delay differential equations exhibit much more complicated dynamics than ordinary differential equations since a time delay could cause a stable equilibrium to become unstable and cause the populations to fluctuate. Recently, a number of researches have been devoted to the dynamic properties for delayed predator-prey systems with harvesting. For further details, refer to [3-7]. 
Kar and Pahari [8] have investigated the dynamical behavior of an exploited system consisting of a prey and a predator that are harvested:

$$
\begin{gathered}
\dot{x}=x(t) g(x(t-\tau))-y p(x(t))-q_{1} E_{1} x(t), \\
\dot{y}=y(t)(-d+\alpha p(x(t)))-q_{2} E_{2} y(t),
\end{gathered}
$$

where $x(t)$ and $y(t)$ respect prey and predator population densities at time $t$ and $g(x)$ is the growth rate of prey when $y(t)$ is zero. $p(x)$ is the response function, $d$ is the death rate of $y(t)$ and $\alpha$ is the conversion factor. $q_{1} E_{1} x$ and $q_{2} E_{2} y$ represent harvesting of $x(t)$ and $y(t)$.

In system (1.1) $x(t)$ and $y(t)$ have the same Holling function. But in real life they may have different adaptation for the environment. So we formulate the aforementioned problem with different Holling functions as follows:

$$
\begin{gathered}
\dot{x}=\alpha\left(1-\frac{x(t-\tau)}{k}\right) x(t)-\frac{\beta x(t) y(t)}{1+b x(t)+c y(t)}-E(t) x(t), \\
\dot{y}=-d y(t)+\frac{a \beta x(t) y(t)}{1+b x(t)} .
\end{gathered}
$$

In 1954, Gordon analyzed the effect of harvest effort on ecosystem from an economic perspective and proposed the following economic theory [9]:

Net Economic Revenue (NER) $=$ Total Revenue $(\mathrm{TR})-$ Total Cost $(\mathrm{TC})$.

Based on the previous economic theory, we let $E(t)$ represent the harvest effort for $x(t)$ at time $t$. TR $=p E(t) x(t)$ and TC $=c_{0} E(t) . p$ represents harvesting reward per unit harvesting effort for unit weight of prey. $c_{0}$ represents the cost per unit harvest effort for prey. Then a differential-algebraic model which consists of two differential equations and an algebraic equation can be established as follows:

$$
\begin{gathered}
\dot{x}=\alpha\left(1-\frac{x(t-\tau)}{k}\right) x(t)-\frac{\beta x(t) y(t)}{1+b x(t)+c y(t)}-E(t) x(t), \\
\dot{y}=-d y(t)+\frac{a \beta x(t) y(t)}{1+b x(t)}, \\
E(t)\left(p x(t)-c_{0}\right)-m=0 .
\end{gathered}
$$

Define

$$
g(x, E)=E(t)\left(p x(t)-c_{0}\right)-m
$$

From the standpoint of biology, we are only interested in the dynamics of model (1.3) in the region

$$
R_{+}^{3}=\{(x, y, E) \mid x \geq 0, y \geq 0, E \geq 0\}
$$




\section{Stability Analysis}

Firstly, we consider the location and number of the equilibria of model (1.3) in $R_{+}^{3}$. The interior equilibrium point is a root of the following equation:

$$
\begin{gathered}
\alpha\left(1-\frac{x_{0}}{k}\right) x_{0}-\frac{\beta x_{0} y_{0}}{1+b x_{0}+c y_{0}}-E_{0} x_{0}=0, \\
-d y_{0}+\frac{a \beta x_{0} y_{0}}{1+b x_{0}}=0, \\
E_{0}\left(p x_{0}-c_{0}\right)-m=0 .
\end{gathered}
$$

It is obvious that (2.1) has an only real solution $Y_{0}=\left(x_{0}, y_{0}, E_{0}\right)$ where

$$
x_{0}=\frac{d}{a \beta-b d^{\prime}}, \quad y_{0}=\frac{\left(\alpha\left(1-x_{0} / k\right)-m /\left(p x_{0}-c_{0}\right)\right)\left(1+b x_{0}\right)}{\beta-c\left(\alpha\left(1-x_{0} / k\right)-m /\left(p x_{0}-c_{0}\right)\right)}, \quad E_{0}=\frac{m}{p x_{0}-c_{0}},
$$

and $\alpha \beta>b d, 0<m<\alpha\left(p x_{0}-c_{0}\right)\left(1-x_{0} / k\right)$. In order to analyze the local stability of the positive equilibrium point for the system (1.3) we use the linear transformation $Y^{T}=Q N^{T}$

$$
N=(u(t), v(t), \bar{E}(t)), \quad Q=\left(\begin{array}{ccc}
1 & 0 & 0 \\
0 & 1 & 0 \\
-\frac{p E_{0}}{p x_{0}-c_{0}} & 0 & 1
\end{array}\right) \text {. }
$$

Then we have

$$
u(t)=x(t), \quad v(t)=y(t), \quad \bar{E}(t)=\frac{p E_{0}}{p x_{0}-c_{0}} x(t)+E(t), \quad D_{y} g(x) Q=\left(0,0, p x_{0}-c_{0}\right),
$$

for which system (1.3) yields

$$
\begin{gathered}
\dot{u}=\alpha\left(1-\frac{u(t-\tau)}{k}\right) u(t)-\frac{\beta u(t) v(t)}{1+b u(t)+c v(t)}-\bar{E}(t) u(t)+\frac{p E_{0} u^{2}(t)}{p x_{0}-c_{0}}, \\
\dot{v}=-d v(t)+\frac{a \beta u(t) v(t)}{1+b u(t)} \\
\left(\bar{E}(t)-\frac{p E_{0} u(t)}{p x_{0}-c_{0}}\right)\left(p u(t)-c_{0}\right)-m=0 .
\end{gathered}
$$

Now we derive the formula for determining the properties of the positive equilibrium point of the system (2.5). Firstly, we consider the local parametric [10] $\psi$, which is defined as follows:

$$
\psi(z(t))=N_{0}^{T}+U_{0} z(t)+V_{0} h(z(t))
$$


where $U_{0}=\left(\begin{array}{ll}1 & 0 \\ 0 & 1 \\ 0 & 0\end{array}\right), V_{0}=\left(\begin{array}{l}0 \\ 0 \\ 1\end{array}\right), z(t)=\left(\begin{array}{l}y_{1}(t) \\ y_{2}(t)\end{array}\right)$, and $h=\left(\begin{array}{l}0 \\ 0 \\ h_{3}\end{array}\right), h_{3}=m /\left(p\left(u_{0}+y_{1}(t)\right)-c_{0}\right)+$ $p E_{0}\left(u_{0}+y_{1}(t)\right) /\left(p x_{0}-c_{0}\right)-E_{0}$. Introducing perturbations $u(t)=u_{0}+y_{1}$ and $v(t)=v_{0}+y_{2}$ and defining $\psi(z(t))=(u(t), v(t), \bar{E}(t))^{T}, \bar{E}(t)=E_{0}+h_{3}\left(y_{(1)}, y_{(2)}\right)$, and $g(\psi(z(t)))=0$, then we can obtain the parametric system of system (2.5) as follows:

$$
\begin{aligned}
\dot{y}_{1}= & \alpha\left(1-\frac{y_{1}(t-\tau)+u_{0}}{k}\right)\left(y_{1}(t)+u_{0}\right)-\frac{\beta\left(y_{1}(t)+u_{0}\right)\left(y_{2}(t)+v_{0}\right)}{1+b\left(y_{1}(t)+u_{0}\right)+c\left(y_{2}(t)+v_{0}\right)} \\
& -\left(\bar{E}_{0}+h_{3}\left(y_{1}(t), y_{2}(t)\right)\right)\left(y_{1}(t)+u_{0}\right)+\frac{p E_{0}\left(y_{1}(t)+u_{0}\right)}{p x_{0}-c_{0}}, \\
\dot{y}_{2}= & -d\left(y_{2}+v_{0}\right)+\frac{a \beta\left(y_{1}(t)+u_{0}\right)\left(y_{2}(t)+v_{0}\right)}{1+b\left(y_{1}(t)+u_{0}\right)} .
\end{aligned}
$$

Neglecting 2nd- and higher-order products of $y_{1}$ and $y_{2}$, we get

$$
\begin{aligned}
\dot{y}_{1}= & \left(\alpha\left(1-\frac{u_{0}}{k}\right)-\frac{\beta v_{0}\left(1+c v_{0}\right)}{\left(1+b v_{0}+c v_{0}\right)^{2}}-E_{0}+\frac{p E_{0} u_{0}}{p x_{0}-c_{0}}\right) y_{1}(t) \\
& -\frac{\alpha u_{0}}{k} y_{1}(t-\tau)-\frac{\beta u_{0}\left(1+b u_{0}\right)}{\left(1+b u_{0}+c v_{0}\right)^{2}} y_{2}(t), \\
\dot{y}_{2}= & \frac{a \beta v_{0}}{\left(1+b u_{0}\right)^{2}} y_{2}(t) .
\end{aligned}
$$

The characteristic equation of system $(2.8)$ at $(0,0)$ takes the form

$$
\lambda^{2}+\left(A-\frac{\alpha u_{0}}{k}+\frac{\alpha u_{0}}{k} e^{(-\lambda \tau)}\right) \lambda+B=0
$$

First of all, we know that the roots of (2.9) with $\tau=0$ have always negative real parts when $A<0, B>0$, where

$$
A=-\alpha\left(1-\frac{2 u_{0}}{k}\right)-\frac{\beta v_{0}\left(1+c v_{0}\right)}{\left(1+b u_{0}+c v_{0}\right)^{2}}-E_{0}+\frac{p E_{0} u_{0}}{p u_{0}-c}, \quad B=\frac{\alpha \beta v_{0}}{\left(1+b u_{0}+c v_{0}\right)^{2}\left(1+b u_{0}\right)^{2}}
$$

Next, we will investigate the distribution of the roots of (2.9). If $i \omega(\omega>0)$ is a root of (2.9), then we have

$$
-\omega^{2}+\left(A-\frac{\alpha u_{0}}{k}+\frac{\alpha u_{0}}{k} e^{i \omega \tau}\right) i \omega+B=0
$$


Separating the real and imaginary parts, we have

$$
\begin{gathered}
\frac{\alpha \omega u_{0}}{k} \sin (\omega \tau)=\omega^{2}-B, \\
\frac{\alpha \omega u_{0}}{k} \cos (\omega \tau)=\omega\left(\frac{\alpha u_{0}}{k}-A\right),
\end{gathered}
$$

which lead to

$$
\omega^{4}+\left[\left(\frac{\alpha u_{0}}{k}-A\right)^{2}-2 B-\left(\frac{\alpha u_{0}}{k}\right)^{2}\right] \omega^{2}+B^{2}=0
$$

When $\left(\alpha u_{0} / k-A\right)^{2}<2 B+\left(\alpha u_{0} / k\right)^{2}$ and $\left[\left(\alpha u_{0} / k-A\right)^{2}-2 B-\left(\alpha u_{0} / k\right)^{2}\right]^{2}>4 B^{2}$ hold, we can get that (2.13) has two positive roots $\omega^{+}$and $\omega^{-}$.

According to the analysis previous, we have the following lemma 2.1:

Lemma 2.1. (1) If $A<0, B>0$ and $\left(\alpha u_{0} / k-A\right)^{2}>2 B+\left(\alpha u_{0} / k\right)^{2}$, then all the roots of (2.9) have negative real parts for all $\tau \geq 0$.

(2) If $\left(\alpha u_{0} / k-A\right)^{2}<2 B+\left(\alpha u_{0} / k\right)^{2}$ and $\left[\left(\alpha u_{0} / k-A\right)^{2}-2 B-\left(\alpha u_{0} / k\right)^{2}\right]^{2}>4 B^{2}$, then (2.13) has two positive roots $\omega^{+}, \omega^{-}$. Furthermore, one has

$$
\tau^{ \pm}=\frac{1}{\omega^{ \pm}} \arccos \left(\frac{k}{\alpha u_{0}}\left(\frac{\alpha u_{0}}{k}-A\right)\right)+\frac{2 n \pi}{\omega^{ \pm}}, \quad n=0,1,2, \ldots
$$

Differentiating the two sides of (2.9) and noticing that $\lambda$ is a function of $\tau$, we have

$$
\left(\frac{d \lambda}{d \tau}\right)^{-1}=\frac{2 \lambda-\left(\alpha u_{0} / k\right) \lambda \tau e^{-\lambda \tau}+\left(A-\alpha u_{0} / k+\left(\alpha u_{0} / k\right) e^{-\lambda \tau}\right)}{\left(\alpha u_{0} / k\right) \lambda^{2} e^{-\lambda \tau}}
$$

and then we can obtain

$$
\operatorname{sign}\left\{\operatorname{Re}\left(\frac{d \lambda}{d \tau}\right)\right\}_{\tau=\tau^{+}, \omega=\omega^{+}}>0, \quad \operatorname{sign}\left\{\operatorname{Re}\left(\frac{d \lambda}{d \tau}\right)\right\}_{\tau=\tau^{-}, \omega=\omega^{-}}<0 .
$$

Therefore, we can obtain the following results about the stability of the positive equilibrium and the Hopf bifurcation of (2.7).

Theorem 2.2. Assume that $A<0, B>0$.

(1) If $\left(\alpha u_{0} / k-A\right)^{2}<2 B+\left(\alpha u_{0} / k\right)^{2}$ and $\left[\left(\alpha u_{0} / k-A\right)^{2}-2 B-\left(\alpha u_{0} / k\right)^{2}\right]^{2}>4 B^{2}$ are satisfied, then there exists an integer $N$ such that the zero solution of system (2.7) is asymptotically stable when $\tau \in\left[0, \tau_{0}^{+}\right) \cup\left(\tau_{0}^{-}, \tau_{1}^{+}\right) \cup\left(\tau_{1}^{-}, \tau_{2}^{+}\right) \cup \cdots \cup\left(\tau_{N-1}^{-}, \tau_{N}^{+}\right)$and unstable when $\tau \in\left(\tau_{0}^{+}, \tau_{0}^{-}\right) \cup\left(\tau_{1}^{+}, \tau_{1}^{-}\right) \cup \cdots \cup\left(\tau_{N^{\prime}}^{+}+\infty\right)$.

(2) When $\tau=\tau_{n}^{ \pm}, n=0,1,2 \ldots$, there are Hopf bifurcations at the equilibrium point of system (2.7). 


\section{Direction and the Stability of Hopf Bifurcation}

In the previous section, we have already obtained the conditions which ensure that model (2.7) undergoes the Hopf bifurcation at the critical values $\tau_{n}(n=0,1, \ldots)$. In this section, we will study the direction and stability of the Hopf bifurcation based on the normal form approach theory and center manifold theory introduced by Hassard et al. [11].

First of all, by the transformation $y_{1}=u-u_{0}, y_{2}=v-v_{0}, t=t / \tau, \tau=\tau_{n}+\mu$, the parametric system (2.7) is equivalent to the following Functional Differential Equation (FDE) system in $C=C\left([-1,0], R^{2}\right)$ :

$$
\dot{z}(t)=L_{\mu}\left(z_{t}\right)+f\left(\mu, z_{t}\right),
$$

where $z(t)=\left(y_{1}(t), y_{2}(t)\right)^{T}, z_{t}(\theta)=z(t+\theta)$ for $\theta \in[-1,0]$, and $L_{\mu}: C \rightarrow R: f: R \times C \rightarrow R$.

$$
\begin{gathered}
L_{\mu}(\phi)=\left(\tau_{n}+\mu\right)\left(\begin{array}{cc}
a_{11} & a_{12} \\
a_{21} & 0
\end{array}\right) \phi^{T}(0)+\left(\tau_{n}+\mu\right)\left(\begin{array}{cc}
b_{11} & 0 \\
0 & 0
\end{array}\right) \phi^{T}(-1), \\
a_{11}=\left(\alpha-\frac{\alpha u_{0}}{k}\right)-\frac{\beta v_{0}\left(1+c v_{0}\right)}{\left(1+b u_{0}+c v_{0}\right)^{2}}-E_{0}+\frac{p E_{0} u_{0}}{p u_{0}-c_{0}}, \\
a_{12}=\frac{-\beta u_{0}\left(1+b u_{0}\right)}{\left(1+b u_{0}+c v_{0}\right)^{2}}, \quad a_{21}=\frac{a \beta v_{0}}{\left(1+b v_{0}\right)^{2}}, \quad b_{11}=-\frac{\alpha u_{0}}{k} .
\end{gathered}
$$

Let $\phi=\left(\phi_{1}, \phi_{2}\right)^{T}$. Then

$$
f(\mu, \phi)=\left(\tau_{n}+\mu\right)\left(f_{11}, f_{22}\right)^{T},
$$

with

$$
\begin{aligned}
f_{11}= & \left(\frac{p E_{0}}{p x_{0}-c_{0}}-\frac{p^{2} E_{0} u_{0}}{\left(p x_{0}-c_{0}\right)^{2}}+\frac{\beta b v_{0}}{\left(1+b u_{0}+c v_{0}\right)^{3}}\right) \phi_{1}(0)^{2}-\frac{\alpha}{k} \phi_{1}(0) \phi_{1}(-1) \\
& -\frac{\beta}{\left(1+b u_{0}+c v_{0}\right)^{2}} \phi_{1}(0) \phi_{2}(0), \\
f_{22}= & -\frac{\beta a b v_{0}}{\left(1+b u_{0}+c v_{0}\right)^{3}} \phi_{1}(0)^{2}+\frac{a \beta}{\left(1+b u_{0}+c v_{0}\right)^{2}} \phi_{1}(0) \phi_{2}(0) .
\end{aligned}
$$

By the Riesz representation theorem, there exists a matrix whose components are bounded variation functions $\eta(\theta, \mu)$ such that

$$
L_{\mu}=\int_{-1}^{0} d \eta(\theta, \mu) \phi(\theta), \quad \phi \in C, \theta \in[-1,0] .
$$

In fact, we can choose

$$
\eta(\theta, \mu)=\left(\tau_{n}+\mu\right)\left(\begin{array}{cc}
a_{11} & a_{12} \\
a_{21} & 0
\end{array}\right) \delta(\theta)+\left(\tau_{n}+\mu\right)\left(\begin{array}{cc}
b_{11} & 0 \\
0 & 0
\end{array}\right) \delta(\theta+1)
$$


where

$$
\delta(\theta)= \begin{cases}0, & \theta \neq 0, \\ 1, & \theta=0\end{cases}
$$

for $\phi \in C^{1}\left([-1,0], R^{2}\right)$. Define

$$
\begin{gathered}
A(\mu) \phi(\theta)= \begin{cases}\frac{d \phi(\theta)}{d \theta}, & -1 \leq \theta<0, \\
\int_{-1}^{0} d \eta(\theta, \mu) \phi(\theta), & \theta=0\end{cases} \\
R(\mu) \phi= \begin{cases}0, & \theta \in[-1,0), \\
f(\mu, \phi), & \theta=0 .\end{cases}
\end{gathered}
$$

Then the system (3.1) is equivalent to

$$
\dot{z}_{t}=A(\mu) z_{t}+R(\mu) z_{t}
$$

for $\psi \in C\left([0,1],\left(R^{2}\right)^{*}\right)$, the adjoint operator $A^{*}$ of $A$ is

$$
A^{*} \psi(s)= \begin{cases}-\frac{d \psi(s)}{d s}, & 0<s \leq 1, \\ \int_{-1}^{0} d \eta^{T}(s, 0) \psi(-s), & s=0\end{cases}
$$

and a bilinear inner product is given by

$$
\langle\psi(s), \phi(\theta)\rangle=\bar{\psi}(0) \phi(0)-\int_{\theta=1}^{0} \int_{\xi=0}^{\theta} \bar{\psi}(\xi-\theta) d \eta(\theta) \phi(\xi) d \xi .
$$

By the discussion in Section 2, we know that $\pm i \tau_{n} \omega^{ \pm}$are eigenvalues of $A(0)$. Thus, they are also eigenvalues of $A^{*}$. It is easy to calculate that

$$
q(\theta)=(1, r)^{T} e^{i \omega \pm \tau_{n} \theta}, \quad q^{*}(s)=G\left(r^{*}, 1\right) e^{i \omega \pm \tau_{n} s},
$$

where

$$
\begin{gathered}
r=\frac{i \omega-a_{11}-b_{11} e^{-i \omega \tau_{n}}}{a_{12}}, \quad \bar{G}=\frac{1}{r+\overline{r^{*}}-\tau_{n} \overline{r^{*}} b_{11} e^{-i \omega \tau_{n}}}, \\
\left\langle q^{*}(s), q(\theta)\right\rangle=1, \quad\left\langle q^{*}(s), \bar{q}(\theta)\right\rangle=0 .
\end{gathered}
$$

Let $z_{t}$ be the solution of (3.9) when $\mu=0$. Define

$$
\dot{z}(t)=\left\langle q^{*}, z_{t}\right\rangle, \quad w(t, \theta)=z_{t}-2 \operatorname{Re}\left\{z_{t} q(\theta)\right\} .
$$


On the center manifold $C_{0}$, we have

$$
w(t, \theta)=w(z(t), \bar{z}(t), \theta)=w_{20}^{(\theta)} \frac{z^{2}}{2}+w_{11}^{(\theta)} z \bar{z}+w_{02}^{(\theta)} \frac{\bar{z}^{2}}{2}+\cdots
$$

Note that $w$ is real. When $\mu=0$ we have

$$
\dot{z}=i \omega \tau_{n} z+\left\langle q^{*(\theta)}, f(0, \omega+2 \operatorname{Re} z(t) q(\theta))\right\rangle .
$$

Then

$$
\dot{z}=i \omega \tau_{n} z+g(z, \bar{z})
$$

where

$$
g(z, \bar{z})=g_{20}(\theta) \frac{z^{2}}{2}+g_{11}(\theta) z \bar{z}+g_{02}(\theta) \frac{\bar{z}^{2}}{2}+\cdots
$$

From (3.9) and (3.16), we have

$$
\dot{W}=\dot{z}_{t}-\dot{z} q-\dot{\bar{z}} \bar{q}= \begin{cases}A w-2 \operatorname{Re} \bar{q}^{*}(0) f(z, \bar{z}) q(\theta), & -1 \leq \theta \leq 0, \\ A w-2 \operatorname{Re} \bar{q}^{*}(0) f(z, \bar{z}) q(\theta)+f, & \theta=0 .\end{cases}
$$

Rewrite

$$
\dot{W}=A W+H(z, \bar{z}, \theta)
$$

where

$$
H(z, \bar{z}, \theta)=H_{20}^{(\theta)} \frac{z^{2}}{2}+H_{11}^{(\theta)} z \bar{z}+H_{02}^{(\theta)} \frac{\bar{z}^{2}}{2}+\cdots
$$

We can obtain

$$
\left(A-2 i \omega \tau_{n}\right) w_{20}=-H_{20}^{(\theta)}, \quad A w_{11}^{(\theta)}=-H_{11}^{(\theta)} .
$$

Now we have $g_{20}, g_{11}, g_{02}$, and $g_{21}$ :

$$
\begin{aligned}
& g_{20}=2 \bar{G} \tau_{n}\left[F \overline{r^{*}}-\frac{\beta r \overline{r^{*}}-a r \beta}{\left(1+b u_{0}+c v_{0}\right)^{2}}-\frac{\alpha \overline{r^{*}}}{k} e^{-i \omega \tau_{n} \theta}-\frac{\beta a b v_{0}}{\left(1+b u_{0}+c v_{0}\right)^{3}}\right], \\
& g_{11}=\bar{G} \tau_{n}\left[2 \overline{r^{*}}+\left(2 a B-2 B \overline{r^{*}}\right) \operatorname{Re}(r)-\frac{2 \beta a b v_{0}}{\left(1+b u_{0}+c v_{0}\right)^{3}}-\frac{2 \alpha \overline{r^{*}}}{k} \operatorname{Re}\left(e^{i \omega \tau_{n} \theta}\right)\right], \\
& g_{02}=2 \bar{G} \tau_{n}\left[F \overline{r^{*}}-\frac{\beta \bar{r} \overline{r^{*}}-a \beta \bar{r}}{\left(1+b u_{0}+c v_{0}\right)^{2}}-\frac{\alpha \overline{r^{*}}}{k} e^{i \omega \tau_{n} \theta}-\frac{\beta a b v_{0}}{\left(1+b u_{0}+c v_{0}\right)^{3}}\right]
\end{aligned}
$$


Journal of Applied Mathematics

$$
\begin{aligned}
g_{21}=2 \bar{G} \tau_{n}[ & \left(2 \overline{r^{*}}-\frac{\alpha \overline{r^{*}}}{k} e^{-i \omega \tau_{n} \theta}-\frac{2 \beta a b v_{0}}{\left(1+b u_{0}+c v_{0}\right)^{3}}+\frac{a \beta r-\beta r \overline{r^{*}}}{\left(1+b u_{0}+c v_{0}\right)^{2}}\right) W_{20}^{(1)}(0) \\
& +\frac{\left(a \beta-\beta \overline{r^{*}}\right)}{\left(1+b u_{0}+c v_{0}\right)^{2}} W_{11}^{(2)}(0) \\
& +\left(F \overline{r^{*}}-\frac{\beta a b v_{0}}{\left(1+b u_{0}+c v_{0}\right)^{3}}-\frac{\alpha \overline{r^{*}}}{2 k} e^{i \omega \tau_{n} \theta}+\frac{a \beta \bar{r}-\beta \bar{r} \overline{r^{*}}}{2\left(1+b u_{0}+c v_{0}\right)^{2}}\right) W_{20}^{(1)}(0) \\
& \left.-\frac{\alpha \overline{r^{*}}}{k} W_{11}^{(1)}(-1)+\frac{a \beta-\beta \overline{r^{*}}}{2\left(1+b u_{0}+c v_{0}\right)^{2}} W_{20}^{(2)}(0)-\frac{\alpha \overline{r^{*}}}{2 k} W_{20}^{(1)}(-1)\right]
\end{aligned}
$$

where

$$
\begin{aligned}
& F=\frac{p E_{0}}{p x_{0}-c_{0}}-\frac{p^{2} E_{0} u_{0}}{\left(p x_{0}-c_{0}\right)^{2}}+\frac{\beta b v_{0}}{\left(1+b u_{0}+c v_{0}\right)^{3}} \\
& W_{20}(\theta)=\frac{i g_{20}}{\tau_{n} \omega} q(0) e^{i \omega \tau_{n} \theta}+\frac{i \bar{g}_{02}}{3 \omega \tau_{n}} \bar{q}(0) e^{-i \omega \tau_{n} \theta}+M_{1} e^{2 i \omega \tau_{n} \theta} \text {, } \\
& W_{11}(\theta)=-\frac{i g_{11}}{\tau_{n} \omega} q(0) e^{i \omega \tau_{n} \theta}+\frac{i \bar{g}_{11}}{\omega \tau_{n}} \bar{q}(0) e^{-i \omega \tau_{n}}+M_{2}, \\
& M_{1}^{(1)}=-\frac{4 A_{1}^{(1)} i \omega+2 a_{12} A_{1}^{(2)}}{a_{21} a_{12}+2 b_{11} i \omega e^{-2 i \omega \tau_{n}}+4 \omega^{2}+2 a_{11} i \omega}, \\
& M_{1}^{(2)}=-\frac{2 A_{1}^{(1)} a_{21}+\left(4 i \omega-2 a_{21}-2 b_{11} e^{-2 i \omega \tau_{n}}\right) A_{1}^{(2)}}{a_{21} a_{12}+2 b_{11} i \omega e^{-2 i \omega \tau_{n}}+4 \omega^{2}+2 a_{11} i \omega}, \\
& M_{2}^{(1)}=-\frac{2 A_{2}^{(2)}}{a_{21}}, \quad M_{2}^{(2)}=\frac{-2 a_{21} A_{2}^{(1)}+2\left(a_{11}+b_{11}\right) A_{2}^{(2)}}{a_{12} a_{21}} . \\
& A_{1}^{(1)}=F-\frac{\beta r}{\left(1+b u_{0}+c v_{0}\right)^{2}}-\frac{\alpha}{k} e^{-i \omega \tau_{n} \theta}, \quad A_{1}^{(2)}=\frac{a \beta r}{\left(1+b u_{0}+c v_{0}\right)^{2}}-\frac{\beta a b v_{0}}{\left(1+b u_{0}+c v_{0}\right)^{3}}, \\
& A_{2}^{(1)}=F-\frac{\beta \operatorname{Re}(r)}{\left(1+b u_{0}+c v_{0}\right)^{2}}-\frac{\alpha}{k} \operatorname{Re}\left(e^{i \omega \tau_{n} \theta}\right), \quad A_{2}^{(2)}=\frac{a \beta \operatorname{Re}(\gamma)}{\left(1+b u_{0}+c v_{0}\right)^{2}}-\frac{\beta a b v_{0}}{\left(1+b u_{0}+c v_{0}\right)^{3}} \text {. }
\end{aligned}
$$

At last, we can calculate

$$
\begin{gathered}
C_{1}(0)=\frac{i}{2 \omega \tau_{n}}\left(g_{11} g_{20}-2\left|g_{11}^{2}\right|-\frac{\left|g_{02}\right|^{2}}{3}\right)+\frac{g_{21}}{2}, \\
\mu_{2}=-\frac{\operatorname{Re}\left\{c_{1}(0)\right\}}{\operatorname{Re}\left\{\lambda^{\prime}\left(\tau_{n}\right)\right\}}, \quad \beta_{2}=2 \operatorname{Re}\left\{c_{1}(0)\right\}, \\
T_{2}=-\frac{\operatorname{Im}\left\{c_{1}(0)\right\}+\mu_{2} \operatorname{Im}\left\{\lambda^{\prime}\left(\tau_{n}\right)\right\}}{\omega \tau_{n}} .
\end{gathered}
$$

Now we have a conclusion. 


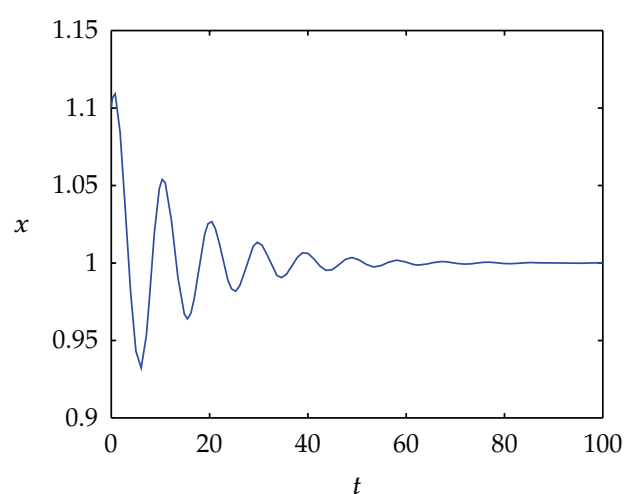

(a)

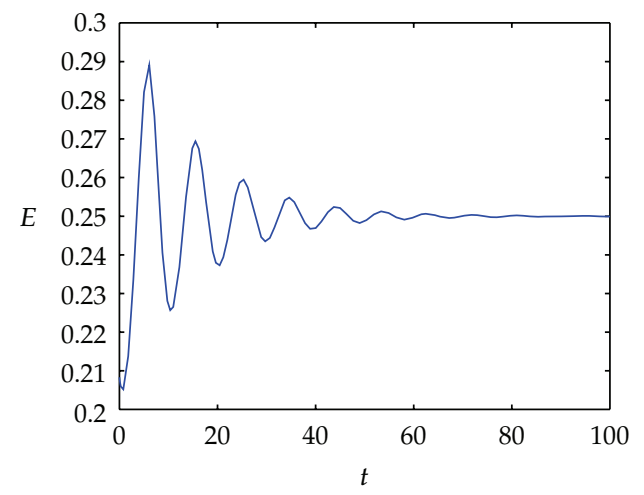

(c)

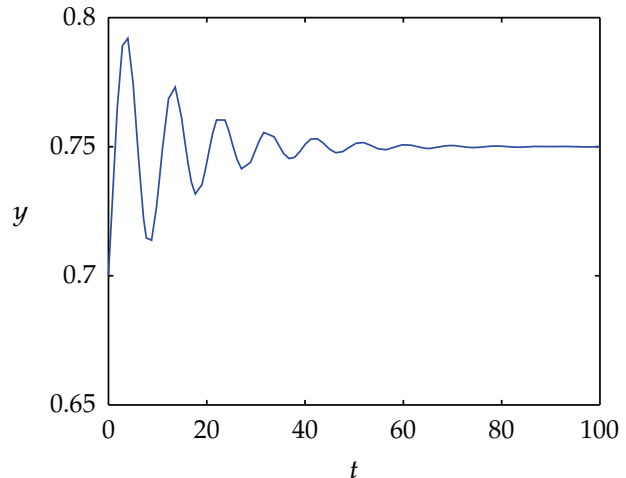

(b)

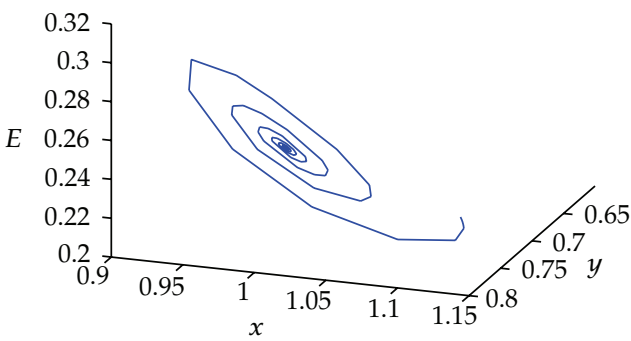

(d)

Figure 1: When $\tau=0.1$, the positive equilibrium point $Y_{0}$ is asymptotically stable.

Conclusion 1. (1) The sign of $\mu_{2}$ determines the direction of Hopf bifurcation. When $\mu_{2}>0$, the Hopf bifurcation is supercritical; when $\mu_{2}<0$, the Hopf bifurcation is subcritical.

(2) $\beta_{2}$ determines the stability of bifurcated periodic solutions. When $\beta_{2}<0$, the periodic solutions are stable; when $\beta_{2}>0$, the periodic solutions is unstable.

(3) $T_{2}$ determines the period of bifurcated periodic solutions. When $T_{2}>0$, the period increases; when $T_{2}<0$, the period decreases.

\section{Numerical Simulations}

In this section, we use some numerical simulations to illustrate the analytical results we obtained in previous sections.

Let $\alpha=\beta=2, k=p=2, a=b=c_{0}=d=1, c=0$, and $m=1 / 4$. The system (1.3) is

$$
\begin{gathered}
\dot{x}=2\left(1-\frac{x(t-\tau)}{2}\right) x(t)-\frac{2 x(t) y(t)}{1+x(t)}-E(t) x(t), \\
\dot{y}=-y(t)+\frac{2 x(t) y(t)}{1+x(t)}, \\
0=E(t)(2 x(t)-1)-\frac{1}{4} .
\end{gathered}
$$




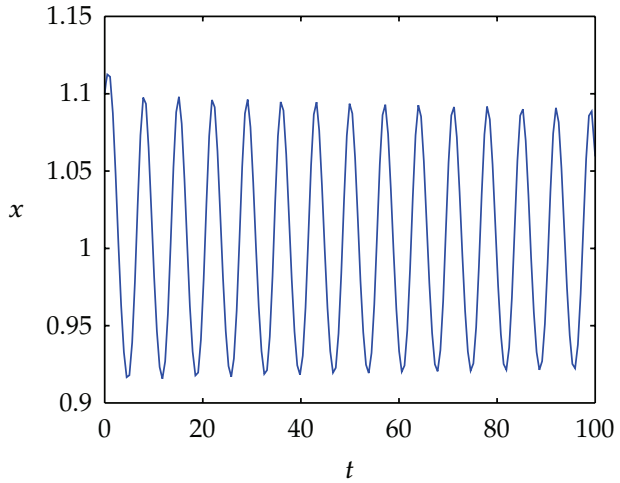

(a)

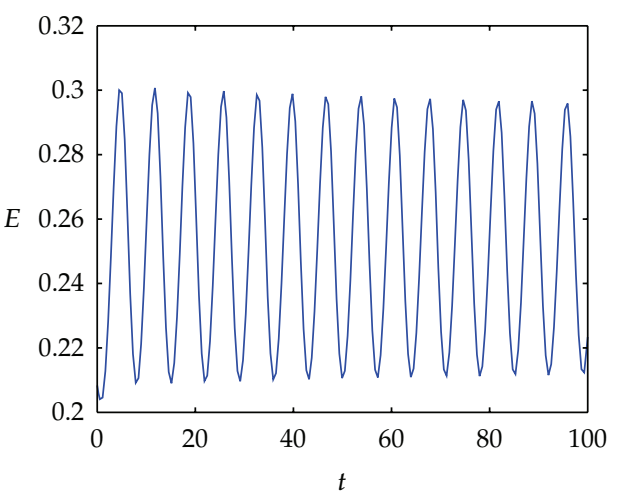

(c)

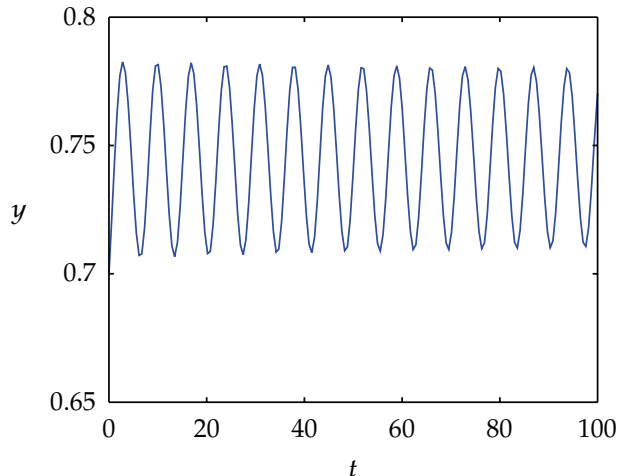

(b)

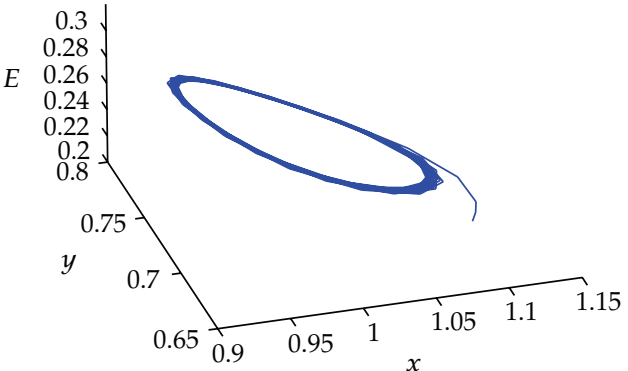

(d)

Figure 2: When $\tau=0.5618$, periodic solutions occur from $Y_{0}$.

The only positive equilibrium point of (4.1) is $Y_{0}=(1,3 / 4,1 / 4)$. By simple computation we have $\omega^{-}=0.4164$ and $\omega^{+}=0.9005$. Substituting these parameters into (2.14) gives $\tau_{0}^{-}=1.2136, \tau_{0}^{+}=0.5612, \tau_{1}^{+}=7.5386$, and $\tau_{1}^{-}=16.2129$. Applying Theorem (2.5), we have that the positive equilibrium point of (4.1) is asymptotically stable when $\tau \in[0,0.5612) \cup(1.2136,7.5386)$. The results can be illustrated in Figures 1 and 3 .

When $\tau>\tau_{0}^{+}=0.5612$ and is sufficiently near $\tau_{0}^{+}$, the bifurcating periodic solution from positive equilibrium point of (4.1) occurs. Furthermore, we consider the properties of the Hopf bifurcation at $\tau=\tau_{0}^{+}=0.5612$. Based on Theorem (2.5) and conclusion 1, we get $c_{1}(0)=0.1673-0.6835 i$, and $\lambda^{\prime}\left(\tau_{0}\right)=0.9563-0.1856 i$. This implies that

$$
\mu_{2}=-0.1749, \quad \beta_{2}=0.3346, \quad T_{2}=1.2883 \text {. }
$$

We conclude that the bifurcating periodic solution is subcritical and unstable. The results are illustrated in Figure 2. 


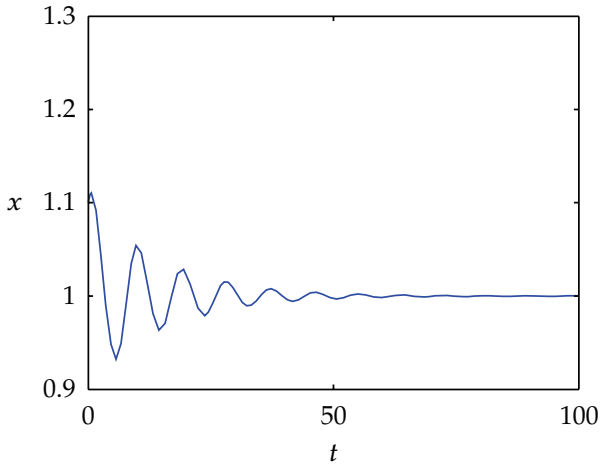

(a)

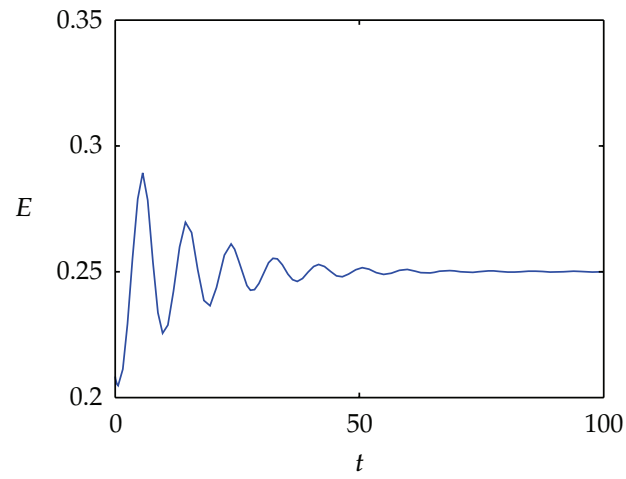

(c)

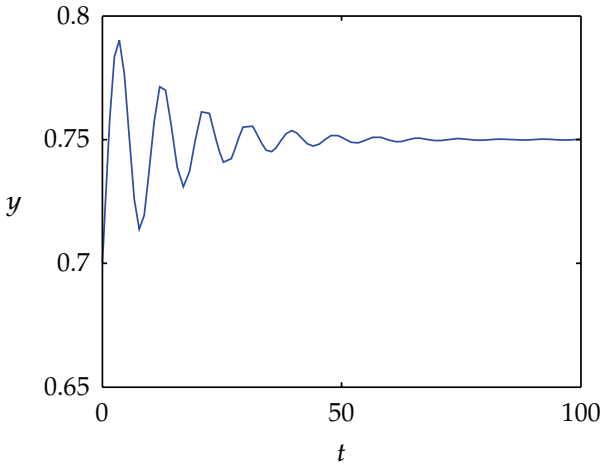

(b)

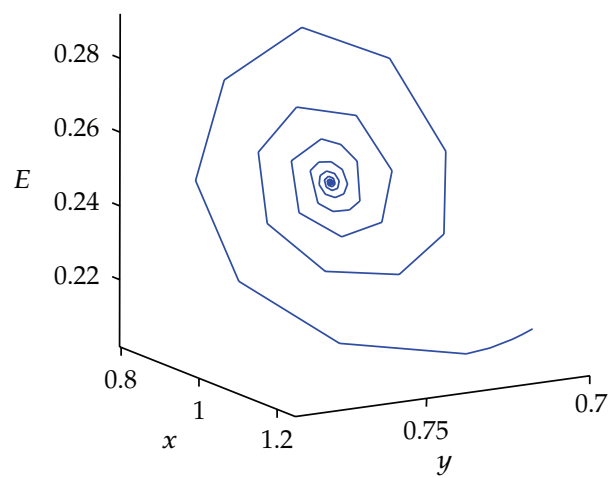

(d)

Figure 3: When $\tau=1.3$, the positive equilibrium point $Y_{0}$ is stable.

\section{Acknowledgment}

This paper was supported by the National Natural Science Foundations of China (10871056).

\section{References}

[1] R. Riaza, "Singularity-induced bifurcations in lumped circuits," IEEE Transactions on Circuits and Systems, vol. 52, no. 7, pp. 1442-1450, 2005.

[2] S. Ayasun, C. O. Nwankpa, and H. G. Kwatny, "Computation of singular and singularity induced bifurcation points of differential-algebraic power system model," IEEE Transactions on Circuits and Systems, vol. 51, no. 8, pp. 1525-1538, 2004.

[3] X.-a. Zhang, L. Chen, and A. U. Neumann, "The stage-structured predator-prey model and optimal harvesting policy," Mathematical Biosciences, vol. 168, no. 2, pp. 201-210, 2000.

[4] T. K. Kar and A. Ghorai, "Dynamic behaviour of a delayed predator-prey model with harvesting," Applied Mathematics and Computation, vol. 217, no. 22, pp. 9085-9104, 2011.

[5] S. A. Gourley and Y. Kuang, "A stage structured predator-prey model and its dependence on maturation delay and death rate," Journal of Mathematical Biology, vol. 49, no. 2, pp. 188-200, 2004.

[6] M. Bandyopadhyay and S. Banerjee, "A stage-structured prey-predator model with discrete time delay," Applied Mathematics and Computation, vol. 182, no. 2, pp. 1385-1398, 2006.

[7] R. Xu, M. A. J. Chaplain, and F. A. Davidson, "Persistence and stability of a stage-structured predatorprey model with time delays," Applied Mathematics and Computation, vol. 150, no. 1, pp. 259-277, 2004. 
[8] T. K. Kar and U. K. Pahari, "Non-selective harvesting in prey-predator models with delay," Communications in Nonlinear Science and Numerical Simulation, vol. 11, no. 4, pp. 499-509, 2006.

[9] H. S. Gordon, "The economic theory of a common property resource: the fishery," Journal of Political Economy, vol. 62, no. 2, pp. 124-142, 1954.

[10] B. S. Chen, X. X. Liao, and Y. Q. Liu, "Normal forms and bifurcations for differential-algebraic systems," Acta Mathematicae Applicatae Sinica, vol. 23, no. 3, pp. 429-443, 2000 (Chinese).

[11] B. D. Hassard, N. D. Kazarinoff, and Y. H. Wan, Theory and Applications of Hopf Bifurcation, Cambridge University Press, Cambridge, UK, 1981. 


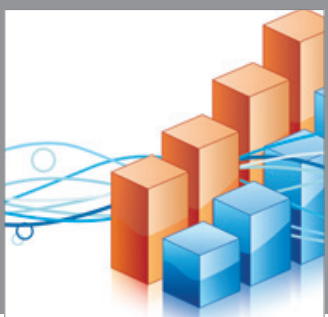

Advances in

Operations Research

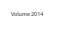

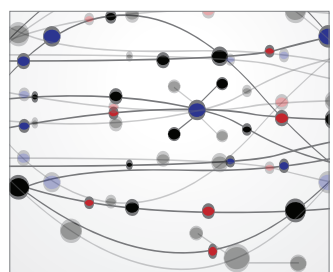

\section{The Scientific} World Journal
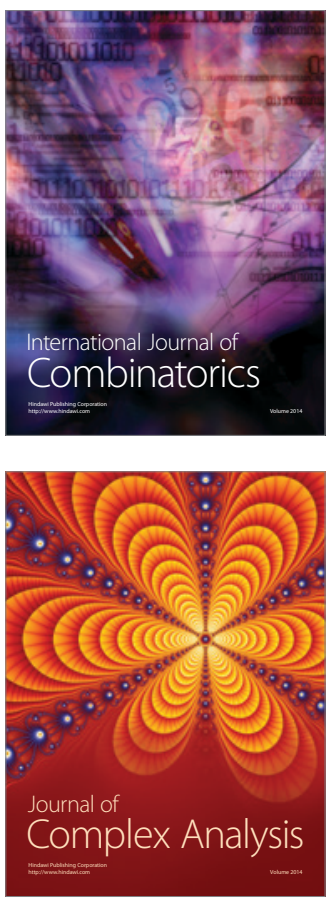

International Journal of

Mathematics and

Mathematical

Sciences
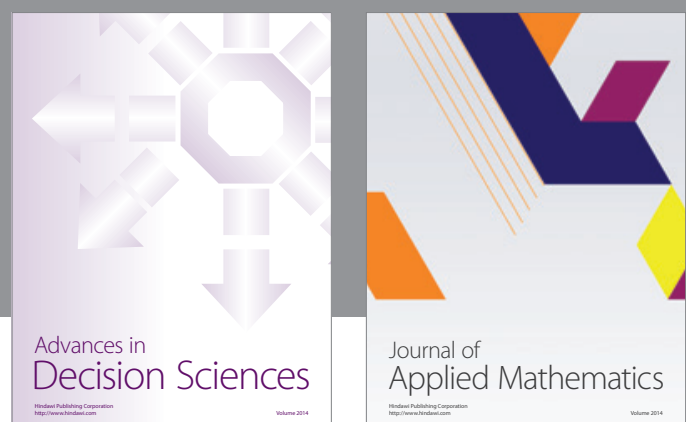

Journal of

Applied Mathematics
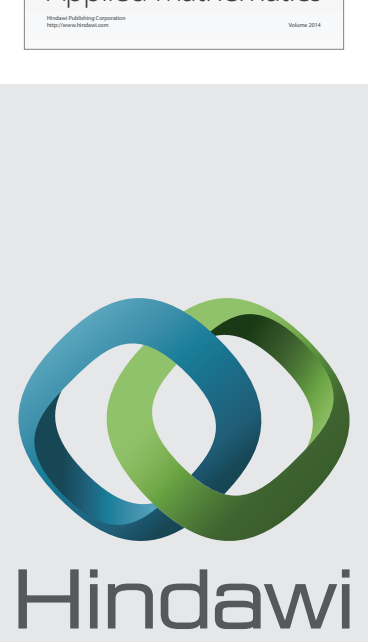

Submit your manuscripts at http://www.hindawi.com
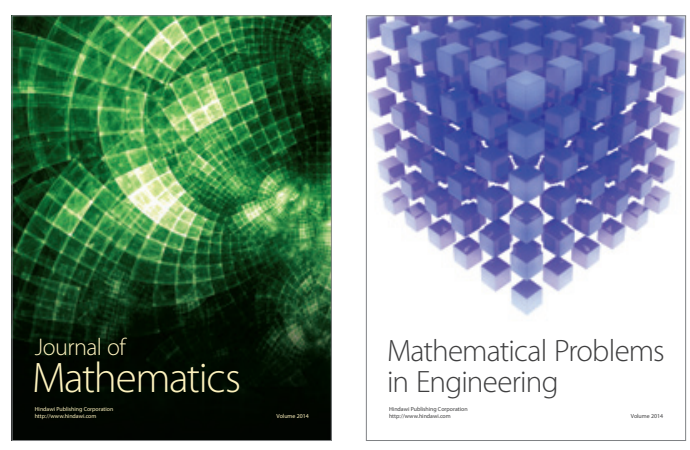

Mathematical Problems in Engineering
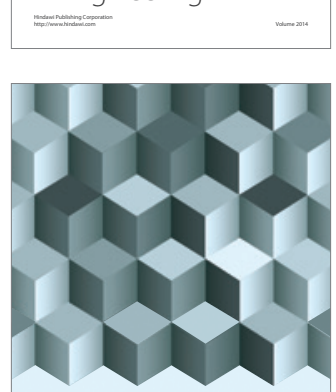

Journal of

Function Spaces
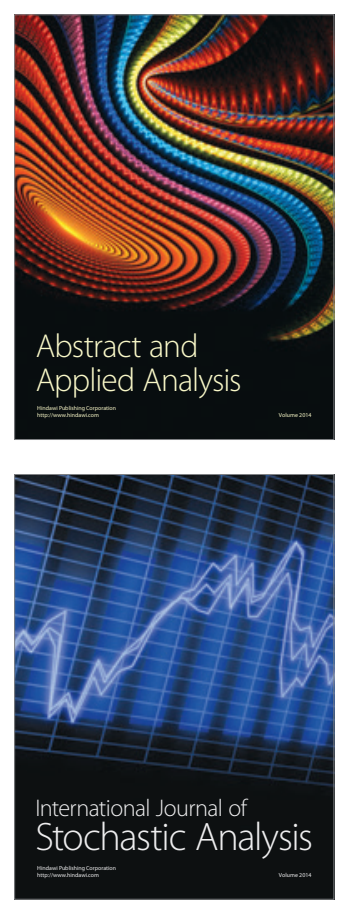

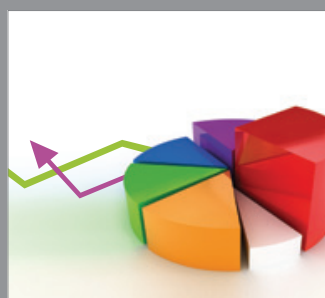

ournal of

Probability and Statistics

Promensencen
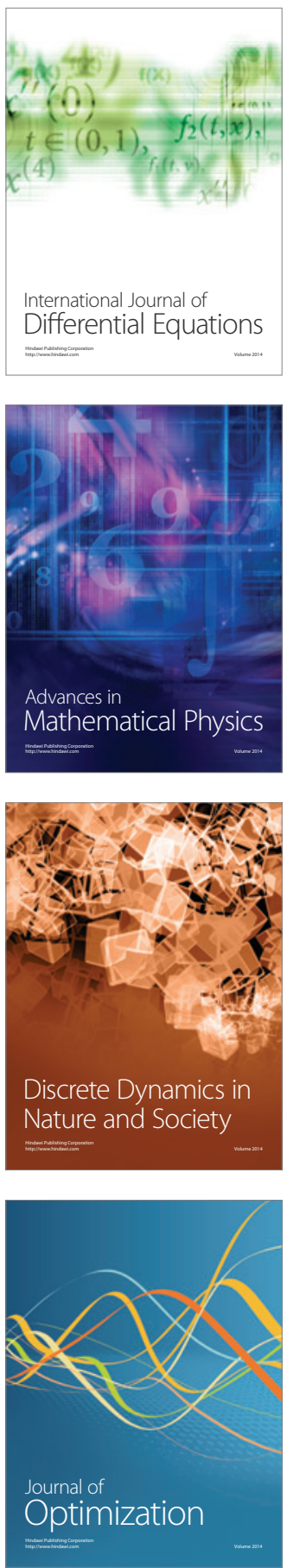